- ACORN Australan college of | JOURNAL OF PERIOPERATIVE NURSING

Volume 31 | Issue 2

Article 3

6-1-2018

\title{
Technology stress in perioperative nursing: An ongoing concern
}

Follow this and additional works at: https://www.journal.acorn.org.au/jpn

Part of the Health Services Administration Commons, Health Services Research Commons, Perioperative, Operating Room and Surgical Nursing Commons, and the Surgery Commons

(c) (i)

This work is licensed under a Creative Commons Attribution 4.0 License.

\section{Recommended Citation}

Smith, Judith and Palesy, Debra (2018) "Technology stress in perioperative nursing: An ongoing concern," Journal of Perioperative Nursing: Vol. 31 : Iss. 2 , Article 3.

Available at: https://doi.org/10.26550/2209-1092.1028

https://www.journal.acorn.org.au/jpn/vol31/iss2/3

This Article is brought to you for free and open access by Journal of Perioperative Nursing. It has been accepted for inclusion in Journal of Perioperative Nursing by an authorized editor of Journal of Perioperative Nursing. 


\section{Authors}

Mrs Judith Smith

MA Nursing, GradCert (Periop Nursing), BN, NE, RN, MACORN, MNSWOTA, MACN, MSTTI Lecturer, Faculty of Nursing, University of Technology - Sydney

Dr Debra Palesy

PhD, MEd, BN, RN, MCHSP

Lecturer, Faculty of Nursing, University of

Technology - Sydney

\section{Corresponding author}

Mrs Judith Smith

Lecturer, Faculty of Nursing, University of

Technology - Sydney

E: Judith.Smith@uts.edu.au

\section{Technology stress in perioperative nursing: An ongoing concern}

Keywords: technology, stress, perioperative, nursing

The following discussion will explore the impact of technology on perioperative nursing roles and the development of the phenomenon coined 'technology stress'. We will begin by reviewing the ongoing debate of technical versus caring nursing practices. The impact of advanced technology on perioperative nursing roles is explored, highlighting the development of technology stress. The paper concludes with some recommendations for further research.

Technology has been an integral part of the surgical environment for decades and continues to develop rapidly, driven by technological innovation. And the integration of multiple and more complex technical systems is very quickly becoming a reality for many perioperative departments. Advanced biotechnology and nanotechnology has seen the emergence of computer-integrated surgery including surgical robots, navigation systems and fully computerised hybrid theatres', with future advances into 3D organ printing and 4D 'augmented reality' including holographic and virtual keyboards soon becoming a reality in the perioperative environment.

In the advanced surgical environment, having expert knowledge about technology is a critical part of providing safe patient care. The perioperative nurse is constantly learning about new equipment and procedures to meet current and future technological challenges.
Perioperative nurses often serve as technology 'super users' responsible for demonstrating quick mastery of these new technologies and sharing this information with their colleagues ${ }^{2}$. However, the pressures of technology mastery, although beneficial to the surgical environment and patient outcomes, has affected perioperative nursing significantly: no sooner do nurses become familiar with one type of technology than it is superseded or upgraded to a more advanced mode ${ }^{3}$. This has resulted in perioperative nurses feeling inadequate in their ability to fulfil their responsibilities, which leads to dissatisfaction in their roles and ultimately develops into stress.

\section{Technician or caregiver?}

There are recognised tensions for perioperative nurses between the nursing philosophy of caring and technological requirements 4 . In 2006 an ethnographic study was conducted in one large Australian hospital operating suite in response to criticism that perioperative nurses are technically focused and as such, do not do real nursing 5 . It was concluded that although the focus of activities is technological, underpinning these activities is an ethic of caring that is evidenced by the centrality of the patient to the nurses' work. Most study respondents described their nursing practice as a blend of 'traditional' care (in terms of interpersonal contact with the patients) and 'technological' ability. However, the study did warn that technology could undermine patient 
care when technological proficiency takes precedence over the provision of care ${ }^{5}$.

Richardson-Tench ${ }^{4}$ further developed these findings in a similar study and found the increased use of technology has created stress for perioperative nursing practice. The results suggest that opportunities for caring in the traditional way are limited in the perioperative environment and have positioned the perioperative nurse as taskorientated, suggesting that '...the powerful discourse of technology is very seductive and that the nursing discourse of caring may be overtaken by the imperative need of the surgical procedure...." (pp. 13-14). It was found that often those with 'technical flair' viewed the patient as more of an object, particularly from a novice nurse perspective, where humanistic caring could not occur until psychomotor skills were mastered ${ }^{4}$.

Another cause for concern is the increasing expectation for perioperative nurses to be competent in creating, storing, finding, manipulating and sharing information (i.e. information technology - IT). As an example, Sweeney ${ }^{6}$ cites the introduction of electronic medical records (EMR). In her exploration of the effects of IT on perioperative nurses' practice, Sweeney found that perioperative nurses felt limited in their IT knowledge and skills, yet many were resistant to training as they believed it was not within their scope of practice, was too time consuming and/or difficult to master. Further, Sweeney expressed concern that increased focus and dependence on technology could reduce human contact with patients.

In overview, perioperative nursing is inextricably linked to technological development and innovation. These advances necessitate ongoing development of technical nursing knowledge and skills, yet there is a real risk of eroding the quality of interpersonal contact and care given to patients ${ }^{3}$. Indeed, the actual notion of 'care' in the perioperative setting is still unclear and remains a challenge?. These competing perspectives may contribute to significant stress as perioperative nurses feel pressured to prioritise technology mastery over traditional care, regardless of their views on nursing care.

\section{Technology and nursing roles}

In the context of technology, the roles and responsibilities of the perioperative nurse becomes somewhat ambiguous, and the question remains unanswered as to how much responsibility perioperative nurses should have for technology in the surgical environment. Luck and Gillespie ${ }^{3}$ suggest as perioperative nursing roles become more techno-centric, nurses are expected to perform routine patient care, in addition to serving as a technician and troubleshooter. This dichotomy is explained by Richardson-Tench ${ }^{\text {, }}$ who proposes that perioperative nurses' practice in an isolated environment where technical expertise and proficiency is awarded more status and prestige than caring and 'nurturing'. However, Bjorn and Bostram ${ }^{9}$ argue that theatre nurses should have a technical focus and responsibility, which includes knowledge of and use of equipment in the operating theatre to ensure patient safety.

Perioperative nurses who are involved in the intraoperative use of technology not only need to be familiar with the specifics of the technology but also the associated instrumentation and surgeon preferences. In the event that the technology or system malfunctions perioperative nurses must be appropriately trained to interpret and correct these errors. How much the surgeon relies on the perioperative nurses to set up, manage and troubleshoot equipment and technology is subjective, and contributes to role ambiguity and technology stress amongst perioperative nurses ${ }^{3}$.

Establishing competency is essential to ensure that perioperative nurses have an understanding of the fundamental knowledge and skills necessary to practice professionally as a registered nurse during procedures using technology in the operating room. However, as Stanton ${ }^{2}$ states there is currently no standardised process for measuring competency with many operating room technologies. The ACORN standards ${ }^{10}$ are the gold standard for perioperative practice in Australia, yet these standards do not provide specific guidelines in relation to the responsibilities of technology in the perioperative environment ${ }^{11}$.

For example, ACORN Anaesthetic nurse role Standard statement 2.6 states that 'The anaesthetic nurse has a duty to, at a minimum, be able to demonstrate core competencies as recommended by the ANZCA PS08 professional standard in relation to ... anaesthesia equipment, ... anaesthesia techniques, ... invasive techniques ... therapeutics ${ }^{10}$ (p.13). The $^{2}$. The ANZCA PS08 ${ }^{12}$ criteria 7.2.1 states the anaesthetic assistant can describe the care, use and servicing of all equipment related to the provision of anaesthesia services including anaesthesia delivery systems and ventilators, monitoring equipment including ultrasound devices, airways devices including fibre optic instruments and intravascular devices. In addition, the ACORN Circulating nurse role ${ }^{10\left(p^{25)}\right.}$ and Instrument nurse role ${ }^{10\left(p^{29}\right)}$ both state that the nurse needs to "be aware 


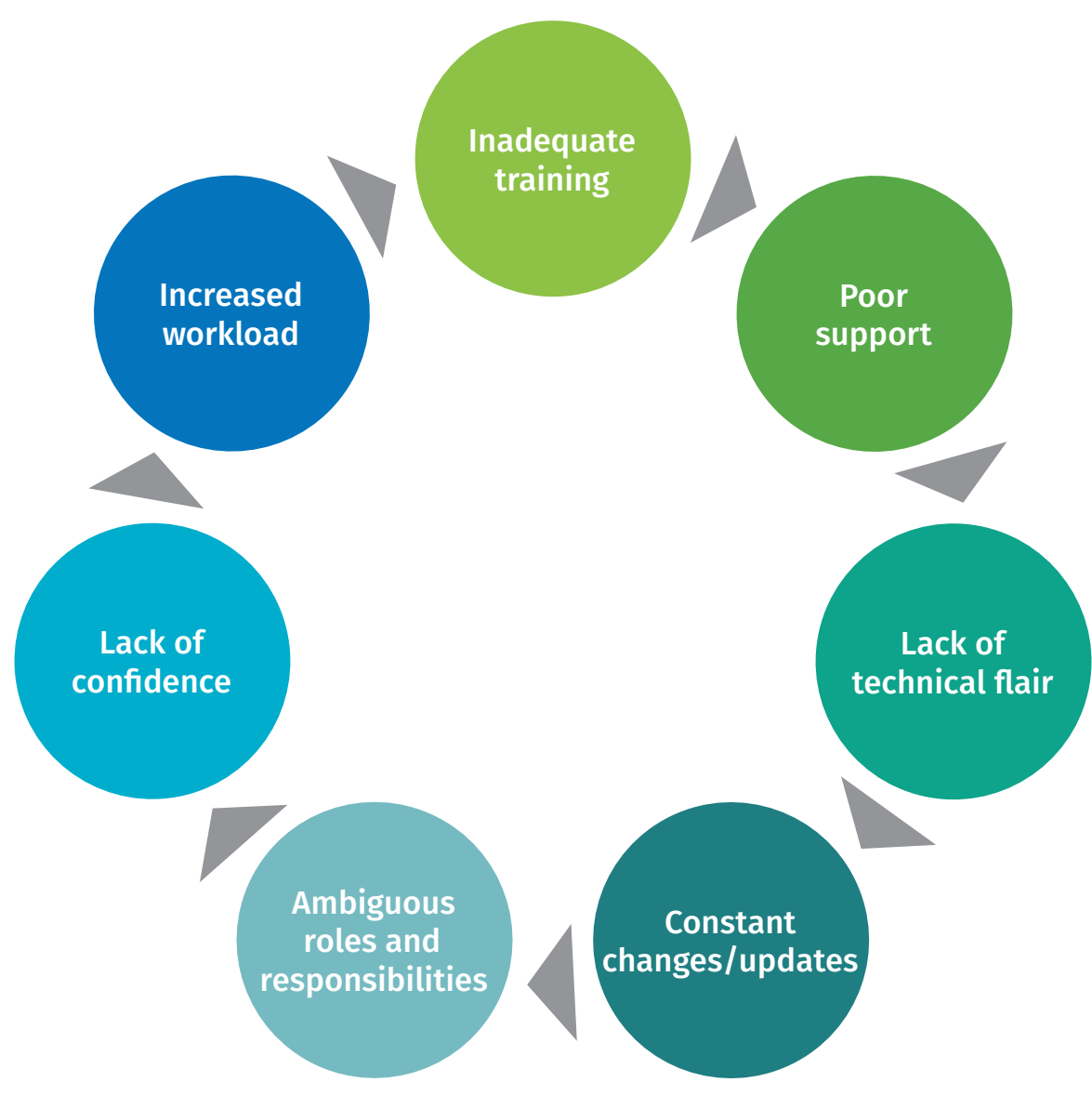

of advances and changes in clinical practice and technology'.

However, these technological 'core competencies' and 'advances and changes' are not specifically defined in the ACORN standards. This may lead to different interpretations between both individual nurses and facilities and create ambiguity and stress for the perioperative nurse. In summary, the scope of responsibility needs to be evaluated and clearly articulated to guide perioperative nurses in their roles related to technology.

\section{Technology stress}

Technology stress for perioperative nurses is not a new phenomenon. With the sudden emergence of multiple surgical technologies in the 1990s, Johnson ${ }^{13}$ first highlighted the competing role demands of the perioperative nurse and subsequent increase in workrelated stress, associating the increased work volume with the technical complexity of the work. Nurses expressed concerns about maintaining quality patient care in an environment characterised by new surgical techniques that were more labour-intensive. Johnson ${ }^{13}$ provided evidence that suggested the development of surgical technologies designed to simplify and streamline surgical techniques had in fact increased work complexity and workload. This contributed to an increase in stress which was attributed in part to role conflict amongst perioperative nurses.

Catalano et al.' had similar findings, stating the introduction of new technologies had caused angst amongst perioperative nurses, even when they had received education and training on the use of the technology. This was due in part to poor understanding of the training provided: either the training was unclear or insufficient. There was also the issue of malfunction or errors with the technology and uncertainty of who was responsible to correct the error, negatively affecting quality care and patient safety. Sweeny ${ }^{6}$ reported perioperative nurses expressing fear, inadequacy or a lack of confidence in their own performance. These fears often interfered with their ability to learn and adapt to new technology. Stanton ${ }^{2}$ emphasised the importance of time for perioperative nurses to gain knowledge and confidence with new technology; however, the reality is that this cannot happen when technology is changing too often.

Sorensen et al.' suggest perioperative nurses fall into two broad categories: those with technical flair and those who lack technical skills. Technical flair is considered to be present when nurses demonstrate skill in carrying out procedures regardless of the amount, size, construction, or variety of different types of equipment and technology. Technical flair involves the ability to easily acquire new knowledge and skills in using instruments and machinery. Alternatively, many perioperative nurses, although able to apply existing knowledge and skills to routine operations, are unable to acquire knowledge and skills in using new instruments or establish routines involving complex technical procedures and computer-based equipment? A lack of technologyrelated skills can have negative consequences on perioperative nurses, including increased workrelated stress, decreased job satisfaction and uncertainty around roles and responsibilities?. Moreover, Sorensen et al. ${ }^{7}$ describe the notion of 'technophobia', where nurses are fearful, clumsy and challenged by technology, and suggest that this may develop in relation to limited technical skills, which further compounds work-related stress. 
Jacobs $^{11}$ also identified an increased use of medical technologies and inadequate training opportunities in the use of these new technologies were factors contributing to perioperative nurses' stress. The author attributes this to the perioperative nurse being expected to be part engineer, part computer technician and part electronic expert on top of specific perioperative nursing skills required to provide quality care for surgical patients.

Findings proposed by Chard ${ }^{14}$, Jacobs $^{11}$ and Vowels et al. ${ }^{15}$ demonstrate the negative impact of stress on the wellbeing of perioperative nurses. Occupational stress can cause perioperative nurses to have physical, psychological, social and spiritual changes such as fatigue, tension, anxiety, fear, anger, depression, feelings of inadequacy, sleep disturbances, burnout and guilt feelings ${ }^{14}$. The development of stress can also impact significantly on staff retention and career progression. A study submitted to the University of Adelaide investigating the recruitment, retention and job satisfaction of perioperative nurses concluded that perioperative nurses in Australia are experiencing moderate to high levels of dissatisfaction in the workplace ${ }^{16}$. Jacobs ${ }^{11}$ discusses stress in relation to decreased job satisfaction and a decrease in quality of care delivered to the surgical patient staff were found to be distracted or overwhelmed and were not able to identify patient needs readily and engage in decision-making processes. Vowels et al..$^{15}$ suggest the pressure to work more quickly and inadequate training to perform tasks, particularly in relation to technology, as the major influences of stress in perioperative nurses.
In summary, literature suggests that a number of compounding factors over the past decades have generated significant technology stress for perioperative nurses. Failure to address these issues has contributed to negative consequences for nurses, their workplaces and ultimately, their patients.

\section{Conclusion and recommendations}

This review proposes that emerging technologies have and will continue to create challenges for perioperative nurses. There is a struggle between the caring and nurturing aspect of nursing and the legitimate need to be competent technicians. This has caused ambiguity and confusion around the roles and responsibilities of perioperative nurses with technology. These challenges have led to the development of 'technology stress' with evidence of significant impact on nurses' wellbeing, health, job satisfaction and retention. This, in turn, has the potential to affect outcomes for the overall perioperative settings and the patients who attend them.

There are implications for further research here. First, we need to better understand the impact of technology on perioperative nurses. This may facilitate the development of a targeted strategy for administrators, educators and perioperative nurse leaders to assist all nurses to manage work-related technology stress. Central to this approach is a review of existing national standards in relation to technology in the perioperative setting with a view to more clearly articulating the roles and responsibilities of perioperative nurses in relation to technology and/ or establishing new guidelines for managing this ongoing concern.

\section{References}

1. Catalano K, Fickenscher K. Emerging technologies in the OR and their effect on perioperative professionals. AORN J 2007;86(6):958-968.

2. Stanton C. Keeping up with technology. AORN J 2011;93(1):1-9.

3. Luck E, Gillespie B. Technological advancements in the OR: Do we need to redefine intraoperative nursing roles? AORN J 2017;106(4):280-282.

4. Richardson-Tench M. Technician or nurturer: Discourse within the OR. ACORN J 2007;20(3):12-15.

5. Bull R, Fitzgerald $M$. Nursing in a technological environment: Nursing care in the operating room. Int J Nurs Pract 2006;12(1):3-7.

6. Sweeney P. The effects of information technology on perioperative nursing. AORN J 2010;92(5):528-540.

7. Sorensen E, Olsen I, Tewes M, Uhrenfeldt L. Perioperative nursing in public hospitals: An ethnography. BMC Nursing 2014;13(45).

8. Richardson-Tench $M$. The scrub nurse: Basking in reflected glory. J Adv Perioper Care 2008;3(154):125-131.

9. Bjorn C, Bostram E. Theatre nurses' understanding of their work: A phenomenographic study at a hospital theatre. J Adv Perioper Care 2008;3(4):149-155.

10. Australian College of Operating Room Nurses Ltd. Standards for Perioperative Nursing in Australia. $14^{\text {th }}$ ed. Adelaide: ACORN, 2016.

11. Jacobs J. Occupational stress of scrub/ scout practitioners: An overview of selected literature. ACORN J 2015;28(3):15-21.

12. Australian and New Zealand College of Anaethetists (ANZCA). PS08 Statement on the assistant for the anaesthetist [Internet]. Melbourne: ANZCA; 2016. Available from: www.anzca.edu.au/documents/ps08-2015statement-on-the-assistant-for-the-anaes.

13. Johnson L. 1990s surgical technologies implicated in role conflict - inducing stress amongst instrument and circulating nurses. ACORN J 2000;13(1):19-27.

14. Chard R. How perioperative nurses define, attribute causes of, and react to intraoperative nursing errors. AORN J 2010;91(1):132-147.

15. Vowels A, Topp R, Berger J. Understanding stress in the operating room: A step toward improving the work environment. Ky Nurse 2012;60(2):5-7.

16. Mewett S. Factors for perioperative nurse retention - an inquiry into the lived experience of perioperative nurses (thesis submission). Adelaide: University of Adelaide, 2013. Available from: digital.library.adelaide.edu.au/dspace/ bitstream/2440/95130/3/02whole.pdf. 